\title{
Oxidative stress in immune cells of adult obese mice fed during their adolescence with a high $(60 \%)$ fat diet
}

\author{
O. Hernández ${ }^{1}$, L. Marín ${ }^{1}$, L. E. Díaz², G. Baccan ${ }^{2,3}$, S. Gómez-Martínez², A. Marcos² \\ and M. De La Fuente ${ }^{1}$ \\ ${ }^{1}$ Department of Physiology. Faculty of Biology, Complutense University of Madrid, ${ }^{2}$ Department of Metabolism and \\ Nutrition. Institute of Food Science, Technology and Nutrition (ICTAN), Spanish National Research Council (CSIC), \\ Madrid, Spain and ${ }^{3}$ Institute of Health Sciences (Federal University of Bahia), Salvador, Brazil
}

\begin{abstract}
Obesity has been proposed as a model of premature immunosenescence. Since oxidative stress is the base of immunosenescence and of the ageing process, the aim of the current study was to find out the redox state of immune cells from obese adult mice fed with a fat-rich diet during adolescence. Young female ICR mice $(n=16)$ (14 weeks old) were divided into two groups: 1$)$ fed ad libitum with a high fat diet (HFD) containing $60 \%$ fat (Harlan), and 2) fed ad libitum with a maintenance diet (Harlan) (Controls). After 14 weeks, the moment in which the animals (28 weeks of age) showed all characteristics of obesity, these mice were sacrificed and peritoneal immune cell suspensions as well as spleen leukocytes were obtained from both groups. In these immune cells the following parameters: xanthine oxidase (XO) (an enzyme that produces oxidants such as the superoxide anion) and catalase (CAT) (an antioxidant enzyme) activities, as well as glutathione (antioxidant) levels were evaluated by using spectrophotometric methods. The results showed that in adult obese mice there was a significant $(P<0.001)$ increase in XO $\left(\mathrm{mU} \mathrm{XO} / 10^{6}\right.$ cells) activity in both spleen and peritoneal immune cells (in spleen: $3.6 \pm 0.5$ vs $2.7 \pm 0.4$, and in peritoneal cells: $3.2 \pm 0.4$ vs $2.3 \pm 0.3$ in obese and control animals, respectively). Nevertheless, these animals showed a significant decrease in the activity of CAT (U/10 ${ }^{6}$ cells) (for example in peritoneal cells: $11 \pm 1$ in obese and $16 \pm 2$ in control mice, $P<0.001$ ), and in the levels of glutathione (nmol/10 $10^{6}$ cells) (for example in spleen: $1.0 \pm 0.2$ in obese and $2.3 \pm 0.7$ in control mice, $P<0.01$ ). In conclusion: adult mice reaching obesity after having been fed with a fat-rich diet during their adolescence show an oxidative stress in the immune system. Since this situation may be related to the impairment of immune cell functions and hence to a shorter life span we could hypothesize that adult obese subjects could have an accelerated ageing and a worse longevity.
\end{abstract}

This work was supported by MICINN (BFU2011-30336), UCM (910379ENEROINN) grants, RETICEF (RD06/0013/0003) from ISCIII and BTSA (The PRONAOS Study, CDTI 2008 1114). 